\title{
Development of a Bayesian Belief Network Runway Incursion Model
}

\author{
Lawrence L. Green \\ NASA Langley Research Center, Hampton, Virginia, 23681
}

\begin{abstract}
In a previous paper, a statistical analysis of runway incursion (RI) events was conducted to ascertain their relevance to the top ten Technical Challenges (TC) of the National Aeronautics and Space Adminis tration (NASA) Aviation Safety Program (AvSP). The study revealed connections to perhaps several of the AuSP top ten TC. That data also identified several primary causes and contributing factors for RI events that served as the basis for developing a system-level Bayesian Belief Network (BBN) model for RI events. The systemlevel BBN model will allow NASA to generically model the causes of RI e vents and to as sess the effectiveness of technology products being developed under NASA funding. These products are intended to reduce the frequency of RI events in particular, and to improve runway safety in general. The development, structure and assessment of that BBN for RI events by a Subject Matter Expert panel are documented in this paper.
\end{abstract}

$\begin{array}{ll} & \\ A C & =\text { Aircraft } \\ A S I A S & =\text { FAA Safety Information Analysis and Sharing } \\ A T A D S & =\text { Air Traffic Activity Data System } \\ A T C & =\text { Air Traffic Control } \\ A v S P & =\text { NASA Aviation Safety Program } \\ B B N & =\text { Bayesian Belief Network } \\ C o m m & =\text { Communications } \\ C P T & =\text { Conditional Probability Table } \\ F A A & =\text { Federal Aviation Administration } \\ F A R & =\text { Federal Aviation Regulation } \\ H F A C S & =\text { Human Factors Analysis and Clas sification System } \\ K E & =\text { Knowledge Elicitation } \\ N A S A & =\text { National Aeronautics and Space Administration } \\ P E D & =\text { Pedestrian } \\ R I & =\text { Runway Incursion } \\ R W & =\text { Runway } \\ S M E & =\text { Subject Matter Expert } \\ T C & =\text { Technical Challenges } \\ V E H & =\text { Vehicle }\end{array}$

\section{Introduction}

$\mathrm{O}$ NE focus area of the National Aeronautics and Space Administration (NASA), enabled through the Aviation Safety Program(AvSP) of the NASA Aeronautics Research Mis sion Directorate, is to improve aviation safety. The AvSP${ }^{1}$ (http://www.aeronautics.nasa.gov/programs_avsafe.htm) seeks to provide increasing capabilities to:

- $\quad$ predict and prevent safety is sues

- monitor for safety is sues in-flight and lessen their impact should they occur

- analyze and design safety is sues out of complex system behaviors

- analyze designs and operational data for potential hazards 
Within this domain, the issue of runway safety is one thrust of investig ation and research. One component of the runway safety thrust is that of runway incursion (RI) events. Runway incursions, as defined by the Office of Runway Safety ${ }^{2}$ of the Federal Aviation Administration (FAA), are the incorrect presence of an aircraft, vehicle or person on the protected area of a surface designated for the landing and take-off of aircraft, as reported by the respective air traffic control personnel.

Looking into the literature on this topic, a recent NASA study on non-towered airports ${ }^{3}$ indicated that the number of RI events is increasing with time, with about half of the events being of low severity and the remainder be ing split among moderate, high, and severe RI events. Among these events, intersecting run ways are noted as the highest contributing factor. A recent presentation by the Boeing Company ${ }^{4}$ shows that flight hours, departures and the size of the world wide fleet have generally increased, while accident rates have remained es sentially flat (but at a very low level) over the last 20 years; the same presentation points to about $6 \%$ of all accidents being as sociated with final approach, landing, takeoff, and initial climb. A recent U.S. Department of Transportation, Volpe Center ${ }^{5}$ report shows that the spacing of parallel runways has just a small effect (if any) on the number of RI events across all severity categories; the same reports illustrates that crossing the hold short line, entering the runway, and crossing a runway as themost likely types of RI events. A recent journal article ${ }^{6}$ illustrates a dramatic increase in the number of RI reported in 2008 compared to previous years, with pilot deviations always being the largest source of these events. A recentFAA report ${ }^{7}$ described the strong correlation among airport geometry, complexity, and various communication tools (including signage and runway markings) with RI events. A Pilots Association report ${ }^{8}$ illustrates the increase in RI events with air traffic, but with overall the RI event being less than 6 per million operations. This report als o points to majordomestic airports (Chicago, Atlanta, Dallas/Fort Worth, Los Angeles, St. Louis and Philadelphia) as having the greatest number of RI events. A paper by Chapman ${ }^{9}$ suggests that pilots may rate the severity of RI events higher than the controllers that report them.

To that end of improving runway s afety, a statistical analy sis ${ }^{10}$ of the Run way Incursion (RI) Database ${ }^{11}$ from the FAA Safety Information Analysis and Sharing (ASIAS) website was conducted to ascertain its relevance to the top ten technical challenges of AvSP. The information contained in the RI database was found to contain data that may be relevant to several of the AvSP ten technical challenges ${ }^{1}$ areas including: 1) the as surance of flight critical systems [i.e., air traffic control (ATC) operations], (2) the dis covery of precursors to safety issues, and 3) improve crew decision-making and response in complex situations.

When combined with other data from the FAA, documenting air traffic volume from calendar years 2000 through 2011, the initial structure of a generic RI event modelemerges. This RI event model enables the study of causes of RI events and assessment of the effectiveness of NASA technology products at various airports and under various environmental and operational conditions. The generic RI event model has been implemented as a Bayesian Belief Network $(\mathrm{BBN})^{12-14}$. Other similar efforts have been recently documented within a group working at NASA $\mathrm{LaRC}^{12,15-17}$.

\section{Model Development}

The NASA Aviation Safety Program(AvSP) conducts cutting-edge research to produce innovative concepts, tools, and technologies that can improve the intrinsic safety attributes of current and future aircraft. The AvSP research centers around a set of Technical Challenges (TCs) that are aligned with program goals and project objectives. These TCs serve to focus research toward solving aviation safety problems and provide a consistent framework to focus, direct, plan, execute, manage, and communicate Center-distributed research. Among the TCs relevant to this work are:

- Assurance of Flight Critical Systems (air traffic control operations)

- Discovery of Precursors to Safety Issues

- Assuring Safe Human-Systems Integration

- Improve Crew Decision-Making and Response in Complex Situations

Several recent BBN modeling efforts ${ }^{12-17}$ have been undertaken to support AvSP portfolio assessments and to determine if the AvSP technologies are addressing/mitigating aviation s afety problems. The characteristics of is sues selected for modeling are:

- A significant accident category based on the historical data and/or future trend

- Alignment with the focus and research areas of AvSP

- Broad coverage on AvSP safety technology products

- Many underlying causal/contributing factors that lead to aviation accidents

- Suitability for a high-level system analysis and modeling 
The modeling philosophy includes the use of a generic, high-level, system-integrated modeling with a systems level risk-based causal model. It should capture the multi-dependencies (interactions) of causal and contributing factors from various problem domains. However, the modeling should not be a representation of a specific accident/incident case, nor a detailed simulation analysis .

In general, the modeling steps undertaken include: 1 ) determining the causalities and cause-to-effect relations based on the historical risks and anticipated future risks from safety data/database and literature reviews, 2) cons tructing a baseline risk-based causal model as a BBN, 3) conducting Subject Matter Experts (SME) Knowledge Elicitation (KE) sessions to review the baseline model structure and to elicit the Conditional Probability Table (CPT) values for the baseline model without product insertions, and 4) inserting the NASA safety technologies/products into the model and eliciting CPT values with products included. The expected modeling results include 1) a quantification of the likelihood/probability of concerned aviation risks, 2) an as sessment of the direct risk mitigation effectiveness of the NASA safety technologies/products, 3) a portfolio gap analys is and 4) a sensitivity analysis for risk drivers.

As noted previously, the Office of Runway Safety of the FAA definition of an RI event is: The incorrect presence of an aircraft, vehicle or person on the protected area of a surface designated for the landing and take-off of aircraft. When this work begun, these RI events were reported by air / ground traffic control personnel in the proper severity category (Cat) defined as:

- $\quad$ Cat $A=$ an accident or near mis s occurred

- Cat $\mathrm{B}=$ significant potential for collision existed

- Cat $\mathrm{C}=$ ample time and/or distance existed to avoid a collision

- $\quad$ Cat $\mathrm{D}=$ an RI event with no immediate safety consequences

- $\quad$ Other $=$ defined subsequently

The FAA now uses the terms: Accident, Cat A (Near Miss), Cat B, Cat C, and Cat D and Other as noted above. To avoid ambiguity for the most important Cat $A$ events, the RI event severity rankings used henceforth in this paper are: Accident, Near Miss, Cat B, Cat C and Other (including Cat D and other from above, mentioned for completeness but this categorization will not be a subject of modeling or expert elicitation). For the purposes of this modeling effort, the scope of attention is restricted to aircraft involved, Cat $C$ and above $R I$ events, with movement restrictions to be defined subs equently. For the purposes of this model, RI events include at least two objects [aircraft (AC), vehicle (VEH) and/or person/pedestrian(PED)] with one of the objects being the aircraft. Not all pos sible combinations of these two objects are of interest to NASA, e.g., VEH in combination with VEH / PED is not a subject of this study. Once initiated, RI events are short in duration and timing is critical; typical landing and takeoff times are 20 to 30 seconds and event severity can easily escalate with just slightly different timing.

In this effort, RI events are modeled in three distinct phases: 1) the RI Event development phase (seconds to hours ) in which the circums tances develop that allow the RI event to occur, 2) the RI Event initiation is the ins tant in time at which Object 1 (AC or VEH / PED considered together) incorrectly appears on therunway (RW), and 3) the RI Event mitigation phase is the 20 to 30 seconds in which one or more mitig ating actions by the involved pilot(s) and or PED / VEH driver may take place.

The initial referenced data base includes $10459 \mathrm{RI}$ events (with no narratives). Among these, seven were accidents, 110 were near misses, 114 were Cat B, 2014 were Cat C, and 3624 were Cat D. Another 4590 RI events in the data base were "Other", listed as Cat E (not meeting the definition of an RI event), N/A (not applicable), or Cat $\mathrm{P}$ (decision pending on categorization). Note that some runway excursion events started as RI events and were categorized as N/A in this database (e.g., August 27, 2006 crash at LEX resulting in 49 deaths - aircraft takeoff on wrong runway $)^{18}$. For events without narratives, this initial data set is useful for es tablishing overall probabilities related to the type of RI events that occur. The final data set consisted of $1596 \mathrm{RI}$ events (Cat C and above, with brief narratives). Of these, there was just one accident (the others excluded because no narrative was provided by the FAA), 30 were near mis ses, 20 were Cat B and 1545 were Cat C events. Of the 1596 RI events, 1299 were caused by AC, 260 were caused by VEH and 37 were caused by PED. The complete data time frame ranges from 2001 through 2011, however, the narrative data time frame ranges from 2007 through 2011. The modeling time frame ranges from 2007 through 2014 or possibly 2015 at the latest.

It is important to realize that RI events are "people intensive", involving possibly two pilots and possibly two controllers (when some form of split control such as air / ground is in effect). A VEH driver or PED could replace one of the pilots. There are also organizations (FAA, Airport Management, etc.) behind each of the people directly involved in the RI event. The Human Factors Analysis and Clas sification System (HFACS) by Wiegmann and Shappe ${ }^{19}$ are frequently used to describe the organizational, supervisory and personal factors states that establish preconditions for human errors and violations. Unfortunately, the narratives provided for the RI events do not 
provide sufficient detail for a standard HFACS as sessment. Hence, within this model, the HFACS states for all of the possible RI event participants are considered together as either being deficient (one or more participant has HFACS is sues, worst case) or fully adequate (no HFACS is sues among any of the participants, best case).

Furthermore, RI events are also "communication (Comm) intensive": several instances of two party communications must simultaneously function properly in order to avoid problems. Two party communications involve both the content and transmis sion of information. The content must be correct and comp lete, timely and not too complex for the situation. The transmis sion must be accomplished without garbled or blocked information exchanges. Instances of two party communications exist between all the involved people in the RI event. Taking this to the next level, split controllers are expected (by cockpit crews) to act as a unified controller and cockpit crews involving a pilot and co-pilot are expected (by control) to operate as a unified ACoperator. Hence, any spilt entity needs adequate internal two party communications and adequate external two party communications must exist between the various entities. Failure of any part of this complex communication network results in deficient two party communications that can lead to confusion, a shared attribute among some or all of the participants.

It is important to understand that the RI event severity rating is based strictly on the time/ distance. The severity rating does not consider the object Federal Aviation Regulations (FAR) vehicle classes, the potential for loss of life or damage to property, the cau sal path or error types, nor does it consider the HFACS states of the participants. In short, the existing FAARI event definitions provide a very narrow way to examine runway safety, especially if the ultimate goal is to study the impact of technology injections. Many interesting questions can be posed and an swered in the context runway safety events that do not directly support RI event modeling by the strict FAA definitions; ans wers to these additional questions would provide significant insight into various aspects of pos sible technology injections and their effectiveness. During this work numerous alternativemodels have been developed, discussed and discarded either because they did not provide a satis factory causal path, or because they were deemed to be too complex for use within the SME elicitation process.

An attempt has been made to restrict the RI event scenarios of interest within this study. Part of this reduced scope involves movement res triction s for the objects involved. Object 1 (AC, VEH or PED) initiates the RI event and must be on the RW at the start of the RI event. Object 1, as an AC, arrived on the RW either by incorrectly landing on it, taxiing onto it, or (in the case of cros sing runways) the AC may be landing or taking off on one RW, while a second $\mathrm{AC}($ Object 2$)$ is using the second RW. If Object 1 is VEH or PED, it is assumed to be an authorized agent of airport (an airport affiliated contractor or employee) that has moved onto the wrong runway or onto the correct runway but at the wrong time. Alternatively, the VEH / PED arrived on the RW by uncontrolled, in appropriate runway access either directly via the airport perimeter, or indirectly through the airport terminal; this case has been excluded fromconsideration. The second aircraft is either on the RW at the start of the RI event, or has just crossed the runway threshold prior to landing.

An RI event perspective versus an aviation perspective has been adopted. This means that every situation considered herein is assumed to result in an aircraft involved, Cat Cor above RI event. Only controlled US airports are considered. TheRI event time frame is as sumed in the range from seconds to minutes. The FAR aircraft types of interest are Part 121 (Commercial) and Part 135 (Air Taxi). These are considered together due to presumed similar equipment levels; this assumption was validated by theSMEpanel. Another aircraft type of interest, and a major contributor to RI events, is Part 91 (General Aviation), though discus sion with the SME panel revealed that virtually any type of aircraft may be operated as a Part 91 vehicle; thus the Part 91 distinction is not very useful in this context. Other categories of AC (military, maintenance taxi) are included in the data, but these are not explicitly of interest in this study. The study considers pilot(s), controller(s), and relevant objects (vehicles and pedestrians) on the ground. The study also indirectly considers various airport geometries, various weather and visibility conditions, and various operating conditions. Thesefactors are considered to be fixed during an RI event, whereas the participant HFACS states and two party communications are considered to be active during an RI event. Likewise, airport signs and markings are considered to be fixed mitigations during the RI event, whereas go arounds, aborted takeoffs or other evasive maneuvers are considered to be active mitigating actions performed by the object operators.

\section{Model Description}

A typicalBBN consists of the model structure and the model content. The model structure consists of a set of relevant definitions, as well as the node names, the node states, the ordering of the defined states for each node to facilitate SME comment, the connecting link topology and the connecting link priority as they enter specific nodes

4

American Institute of Aeronautics and Astronautics 
(again, to facilitate SMEcomment). The model content consists of the sets of marginal and Conditional Probability Table (CPT) values. During the first phase of a typical BBN development cycle, NASA researchers develop (based upon database and literature search) and propose a model structure to an SMEpanel; the development step may take months to complete. Then, the various elements of the proposed structure are reviewed, modified and validated by the SMEpanel. Once the model structure has been agreed upon and validated by the SME panel, a CPT elicitation process (model population) is conducted by a facilitator on behalf of NASA to determine the appropriate model content. Some portions of the model review, modification, validation and population can be conducted in parallel. Once the model has been populated, it is executed to obtain a set of baseline results and a baseline sensitivity analysis.

At this point, a preliminary BBN RI event model has been developed. An SME panel consisting of four consultants was assembled to review the model structure and to populate its content. The SME panel included two pilots and two other aviation expert consultants. The SME panel met over two days for about 14 hours of discussion about the complex RI event problem. A preliminary baseline RI model was agreed upon, as shown in Figure 1; some of the model population was also accomplished; this is not discussed herein. The nodes in Figure 1 are color coded to indicate associations among the various nodes. Generally, the flow of specific contributing factors through causal paths is fromleft to right in the figure. Many items funnel together through the two nodes identified as Fixed and Active Contributing Factors. The node identified as "RI Event Initiation" can be thought of as the start of the active mitigation phase of the RI event, which also ties back to the contributing factors.

The SMEpanel validated many of the proposed definitions and most of the proposed model structure. However, the SME panel also provided significant clarification of several es sential definitions within the RI event model. Moreover, the SMEpanel suggested several structural changes to the model, especially as related to the best way to model the active mitigation phase of the RI events. The overall complexity of the proposed RI event model was reduced from 39 nodes and a combined conditional probability table (CPT) size of 1041 elements to one of 37 nodes and combined CPT size of 735 elements. The remainder of this section describes the current preliminary RI event model. Limited clarifying information is presented about each of the nodes because of the remaining possibility that they may still change. The node name for each is presented along with some clarifying comments. Most of the nodes are binary, meaning they have only two possible states: yes or no; where more states are present in a node, this will be made clear from the explanation. The goal of the SMEelicitation is to provide probabilities for each of the possible states; for example, for the node "Airport Layout", the SMEgoal is to determine the probability of that the Airport Layout is an is sue or not in the RI event.

Starting with the yellow nodes of Figure 1 (lower left hand corner), the node descriptions of the preliminary RI event model follow:

- Airport Layout: The airport layout is an is sue. This may includepotentially confusing elements such as parallel runways (with spacing of less than 1000 feet), intersecting runways, taxiways parallel to and near runways, numerous taxiways crossing runways instead of perimeter taxiways.

- Signs, Markings and Equipment: The signs, markings and/or fixed equipment (e.g., lights) at the airport are deficient.

- Average Traffic Volume: The average traffic volume at this airport, at this time, compared to other airports, is an is sue. For example, if the average traffic volume is high, it may cause a significantly increased work load for controllers and/or pilots; if low, it may result in extended periods of inactivity for controllers.

- Non-Visibility Area for ATC: Areas of the airport not ever visible to controllers is an issue.

- Other Airport Issues: Other is sues that have not been enumerated within this grouping are present. This may include inappropriate levels of security for the terminal and/or airport perimeter that may lead to unauthorized vehicles or pedestrians on the runway surface.

- Airport Issues: One or more of the issues within this grouping are present.

- Visibility: Conditions such that temporarily poor visibility (e.g., fog) is an issue.

- Rain, Snow or Ice: Runway or taxiway contamination from precipitation is an issue.

- Other Weather Issues: Other is sues that have not been enumerated within this grouping are present. This may include lightning, smoke or other factors.

- Weather Issues: One or more of the is sues within this grouping are present.

- Local and National ATC Procedures: The use of ambiguous or non-standardized ATC procedures is an issue.

- ATC Staffing Levels: The staffing level and/or work load management not appropriate for the situation is an issue. 
- Similar Call Signs on Airport Environment: The presence of similar sounding, or similar looking, call signs on the airport environment is an is sue.

- Memory Aids: The lack of standardization related to the use of memory aids for controllers (e.g., devices to remind the controller about a truck on RW) is an is sue.

- Other Operational Issues: Other is sues that havenotbeen enumerated within this grouping are present. This might include communication frequency congestion, too many ACon the same frequency at a given time, training operations in progress, etc.

- Operational Issues: One or more of the is sues within this grouping are present.

- Fixed Contributing Factors: One or more of the following is sues is present: Airport Is sues, Weather Is sues or Operational Is sues. It is as sumed that Fixed Contributing Factors do not change during the RI event.

- Completeness / Correctness: The completeness or correctness of communicated information is an issue. This may include the lack of a required usage for a call sign.

- Timeliness: The timeliness of information transmitted is an issue. Information may not have been transmitted at the appropriate time, i.e., it was not delayed. An assumed part of this may be that any follow-up action required based on the information communicated was alsocompleted in a reas onable and timely manner.

- Complexity: The complexity of information transmitted is an issue. An example from the RI event narratives of too much complexity was one set of several ins tructions trans mitted together from controller to pilot that was confusing for this author even with the airport map being examined while reading the transmitted instructions.

- Comm Content Error: One or more of the issues within this grouping are present.

- Communications Transmission Error: Comm transmis sion is an is sue. This may occur when the Comm systemfails to operate as expected and may include blocked ("stepped on" communications where one party cuts off the communications of another), partially blocked (garbled or inaudible Commtrans mis sion), hardware limitations / malfunctions and/or faulty headset jacks or connections.

- Two Party Comm Error: A Communications ContentError or a Communications Transmis sion Error has resulted in a Two Party Communications Error and is an issue.

- Participant HFACS Issues: The Organization, Supervisory or Personal Factors states of one or more of the participants is an issue.

- Active Contributing Factors: One or more of the following is sues is present: Two Party COMM Error or Participant HFACS Is sues. It is as sumed that Active Contributing Factors may change during the RI event.

The preceding discussion covers all the nodes on the left hand side of Figure 1. These are all the is sues potentially present that enable the RI Event to occur. The nodes and states on the right hand side of the figure generically define a specific RI event, of which numerous types and combinations may occur. NASA would hope to be in a position to broadly address many, if not all, of these specific RI event types with technology injections. Starting with the cyan nodes of Figure 1 (middle bottom), the node descriptions follow:

- Operator Error: The operator of Object 1 (Pilot or VEH Driver / PED) committed an error that initiated the RI event. The most common types of Operator Error are failure to hold short of a runway or using the wrong runway.

- Controller Error: The controller committed an error that initiated the RI event. The most types of Controller Error are a loss of oversight (the controller forgot about something under their control) or a Comm Content Error (e.g., the controller transmitted incorrect or incomplete information)

- Mechanical Failure: A mechanical system(brakes, propulsion or controls) of Object 1 failed to work as expected resulting in an RI event. A mechanical failure for a PED on the runway might be a broken leg.

- AC Present: The RI event has been initiated by the incorrect presence of an aircraft (Object 1) on the protected area of a surface designated for the landing and take-off of aircraft.

- VEH / PED Present: The RI event has been initiated by the incorrect pre sence of a vehicle or pers on (Object 1) on the protected area of a surface designated for the landing and take-off of aircraft.

- RI Event Initiation: An RI Event has been initiated or not.

- Separation Distance: At the start of the RIEvent, Object 1 and Object 2 are separated by a maximum distance of one runway length as defined in Figure 2. It is unders tood that some runways are shorter and others longer. It is assumed the length of the runway in question is appropriate for the 
capabilities/limitations of the aircraft involved. Figure 2 illustrates some of the pos sible combinations of positions and orientations for Object 1 and Object2. Object 1 (highlighted in red) is AC, VEH or PED incorrectly on the run way in question; it may be stopped, moving along the runway or cros sing the runway in question. Object 1 can close to, or distant from, Object 2 (relatively speaking). Object 2 (highlighted in blue) is assumed to be an involved aircraft that has either just crossed the runway threshold (stillin the air) in the process of landing or it is already on the runway at the start of the RI event. Whether Object 1 is on the runway, per se, or has just crossed the hold short line to the side of the runway, the path intersection point is defined to be on the runway in question.

$\circ \quad$ State $=$ Close: Object 1 is separated fromObject 2 by a distance of $1 / 3$ or les s of the run way in question.

- State $=$ Medium: Object 1 is separated from Object 2 by a distance of from $2 / 3$ or less to more than $1 / 3$ of the runway in question.

- State = Far: Object 1 is separated from Object 2 by a dis tance of fromone full runway length or less to more than $2 / 3$ of the runway in question. The SME panel noted that if Object 2 (an approaching aircraft) has not yet crossed the threshold of the runway, there is sufficient distance and reaction time such that the RI Event should not be considered as Cat $\mathrm{C}$ or above.

- Rate of Closure: The sum of the opposing velocity components along the runway in question, as defined in Figure 2. It is as sumed Object 2 has a velocity component primarily aligned with the runway in question. The contributing velocity of Object 1 to the Rate of Closure can be computed from trigonometric considerations of its path geometry, relative to the runway in question, and its velocity magnitude; thus if Object 1 is moving perpendicular to the runway in question, it contributes a zero velocity component to the Rate of Closure.

$\circ \quad$ State $=$ Fast: The rate of closure is in excess of 140 knots.

- State $=$ Medium: The rate of closure is between 80 and 140 knots.

- State = Slow: The rate of closure is less than 80 knots.

- Reaction Time: The amount of time available for possible mitigating actions once an RI event has been initiated.

○ $\quad$ State $=$ Short: eight seconds or les s

- State $=$ Medium: nine to twelve seconds

- State = Long: more than twelve seconds

- Mitigation Phase Error: An additional second error by an in volved participant (the Object Operator or Controller) has occurred after the RI event has been initiated.

- Active Mitigation Effectiveness: The degree of impact that applied mitigating actions (this includes Go-Arounds, Aborted Takeoffs and OtherEvasive Maneuvers on the part of the Object 1 or Object 2 Operators, whether commanded by a Controller or not) had upon the expected RI event severity outcome. Implied in this definition is an as sumption that an SME can establish an expected RI event severity based upon the Rate of Closure and the Separation Dis tance and a second assumption that some determination of the mitigation effectiveness can be made based upon on the Reaction Time available and whether or not a Mitigation Phase Error has occurred.

- State = Ineffective: the applied mitigating actions had no effect upon the expected RI event severity outcome; this is the same degree of impact that having applied no mitigating actions would have had upon the expected RI event severity outcome.

- State = Partially Effective: as a result of the applied mitigating actions, a collision was avoided or a one-step reduction in the expected RI event severity was achieved

○ State = Fully Effective: as a result of the applied mitigating actions, a near mis s was avoided or a two-step reduction in the expected RI event severity was achieved

- Final RI Event Severity: The RI event severity as would be reported by the FAA, including the impact of Contributing Factors and Mitigating Actions is established here. As in the definitions provided earlier, this severity ranking here is based upon an SME asses s ment of time and distance. However, because of the structure of the preliminary RI event model, other factors that may come into consideration during an official RI event clas sification meeting are present in the background of this model. 


\section{Conclusions}

A preliminary Bayesian Belief Network (BBN) model for Runway Incursion (RI) events has been developed. Numerous considerations surrounding the process of developing the preliminary model have been documented in this paper. The proposed RI event model was thoroughly reviewed by a Subject Matter Expert (SME) panel. Numerous changes to the model structure (definitions, node names, node states and the connecting link topology) were suggested by the SMEpanel. The structural details of the resulting BBN model for RI events have also been documented within this paper.

\section{Ack nowledgments}

The author would like to acknowledge the contributions of Dr. Ann Shih, Dr. Ersin Ancel, Dr. Jim Luxhoj for their material contributions to this effort. Their comments and efforts enabled the preliminary model structure to be defined. The author would like to acknowledge the significant contributions of the SME panel to this effort. Their experience, insights and suggestions made this preliminary RI event model clearer, more streamlined and more defensible.

\section{References}

${ }^{1}$ A viation Safety Program Website: http://www.aeronautics.nasa.gov/programs_avsafe.htm, accessed (2/11/2013). ${ }^{2}$ The FAA Runway Incursion database website description: http://www.asias.faa.gov/portal/page/portal/ASIAS_PAGES/LEARN_ABOUTS/RunwayIncursionLearnAbout.pdf (accessed 2/11/2013).

${ }^{3}$ Runway Transgressions at Non-Towered and Tower Closed Airports, NASA Aviation Safety Reporting System (ASRS), Publication 61, July 2003.

${ }^{4}$ The Boeing Company, Statistical Summary of Commercial Jet Airplane Accidents, 2012.

${ }^{5}$ Cardosi, K., Chase, S., and Eon, D., "Runway Safety", U.S. Department of Transportation, Volpe Center, Cambridge, MA, Feb. 12, 2010.

${ }^{6}$ Torres, K. R., Metscher, D. S., and Smith, M., "A Correlational Study of the Relationship bet ween Human Factor Errors and the Occurrence of Runway Incursions", International Journal of Professional Aviation Training \& Testing Research, Vol. 5, (1) (C) 2011 Published by Professional Aviation Board of Certification.

${ }^{7}$ The Federal Aviation Adminis tration, "Wrong Runway Departures", Aviation Safety Information Analysis and Sharing (ASIAS), Office of Aviation Safety, AVS-4 July 2007.

${ }^{8}$ Air Line Pilots Association International, "White Paper: Runway Incursions A Call to Action”, March 2007.

${ }^{9}$ Chapman, R., "Runway Incursions 2000-201: Is Safety Improving?", East Michigan University, Retrieved from http://www.miqrogroove.com/writing/runway-incursions/fulltext.pdf, Copyright @ 2010 by Robert Chapin .

${ }^{10}$ Green, Lawrence L., "Analysis of Run way Incursion Data”, AIAA-2013-4400, 13th AIAA Aviation Technology, Integration, and Operations (ATIO) Conference, Los Angeles, CA, 2013.

${ }^{11}$ The FAA Runway Incursion database website: http://www.asias.faa.gov/portal/page/portal/asias_pages/asias_home/datainfo:databases:p-z(accessed 2/11/2013).

${ }^{12}$ Second Workshop on the Investigation and Reporting of Incidents and Accidents, IRIA 2003, NASA/CP-2003212642, Compiled by Kelly J. Hayhurst and C. Michael Holloway, Langley Research Center, Hampton, Virginia, September 2003.

${ }^{13}$ Luxhøj, J. T., Shih, A. T., Jones, S. M., Ancel, E., and Reveley, M. S., "Safety Risk Knowledge Elicitation in Support of Aeronautical R\&D Portfolio Management: A Case Study," American Society for Engineering Management (ASEM) 33rd International Annual Conference, Virginia Beach, VA, Oct 17-20, 2012.

${ }^{14}$ Luxhøj, J. T., Jalil, M., and Jones, S. M., "A Risk-Based Decision Support Tool for Evaluating Aviation Technology Integration in the National Airspace System," Proceedings of the AIAA's 3rd Annual Aviation Technology, Integration and Operations (ATIO) Technical Forum, Denver, CO, November, 17-19. 2003.

${ }^{15}$ Luxhøj, J. T., "Probabilistic Causal Analy sis for System Safety Risk Assessment in Commercial Air Transport," Proceedings of the Workshop on Investigating and Reporting of Incidents and Accidents (IRIA), Sept 16-19, Williams burg, VA, p.17 NASA/CP-2003-212642, 2003.

${ }^{16}$ Ancel, E., and Shih, A.T., "The Analy sis of the Contribution of Human Factors to the In -flight Loss of Control Accidents," 12 th AIAA Aviation Technology, Integration, and Operations (ATIO) Conference, Indianapolis, IN, Sept 17-19, 2012. 
${ }^{17}$ Shih, A. T., Ancel, E., and Jones, S. M., "Object-oriented Bayesian Networks (OOBN) for Aviation Accident Modeling and Technology Portfolio Impact Assessment," American Societyfor Engineering Management (ASEM) 33rd International Annual Conference, Oct 17-20, 2012.

18“Attempted Takeoff From Wrong Runway Comair Flight 5191, Bombardier CL-600-2B19, N431CA, Lexington, Kentucky, August 27, 2006" National Transportation Safety Board ACCIDENT REPORT NTSB/AAR-07/05, PB2007-910406.

${ }^{19}$ Wiegmann, Douglas A. and Shappell, Scott A., A Human Error Approach to Aviation Accident Analysis, Ashgate Publishing Limited, Burlington VT, 2003. (also, www.hfacs.com, accessed Nov. 14, 2013) 


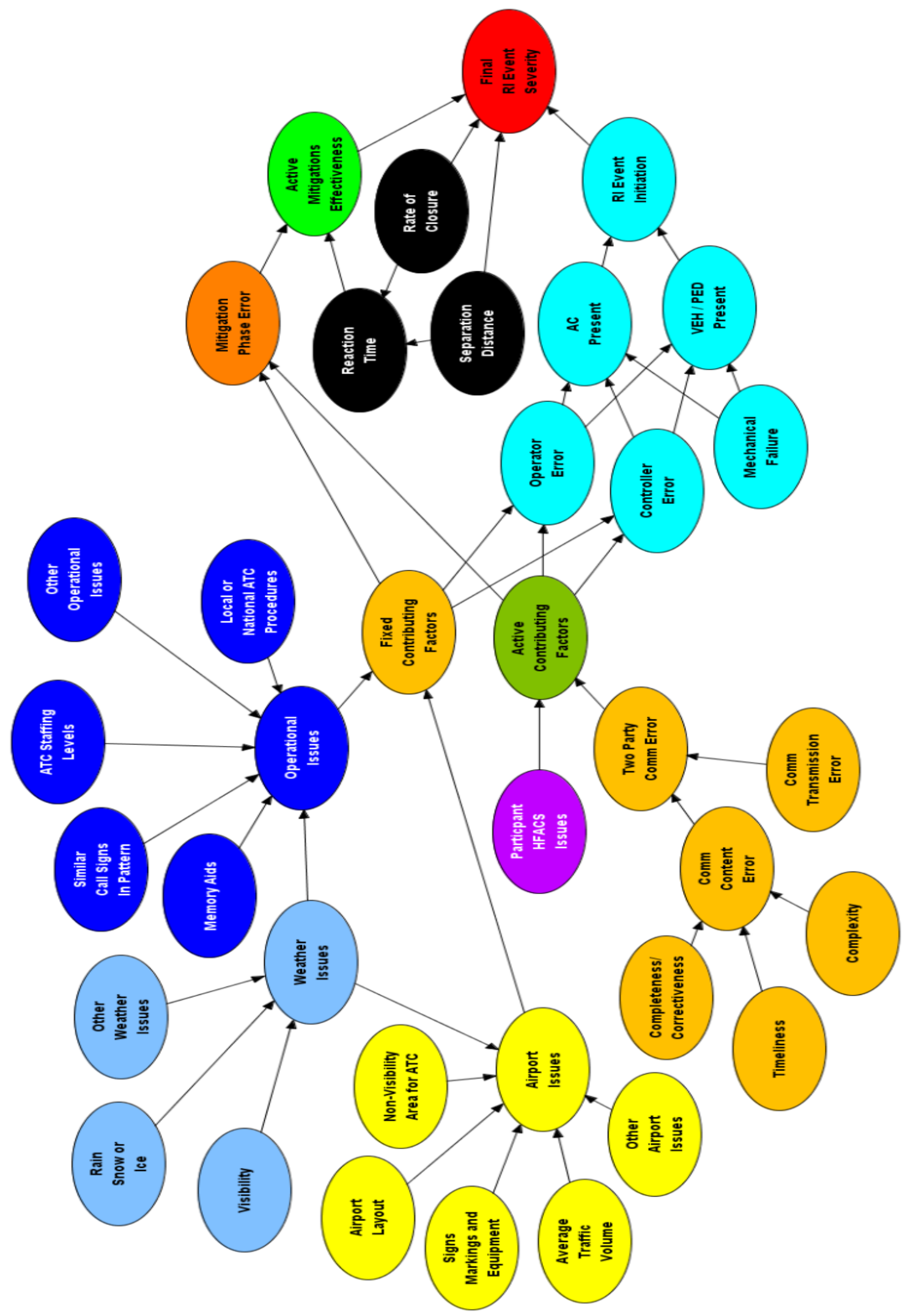

Figure 1. Runway Incursion Bayesian Belief Network Preliminary Model. 


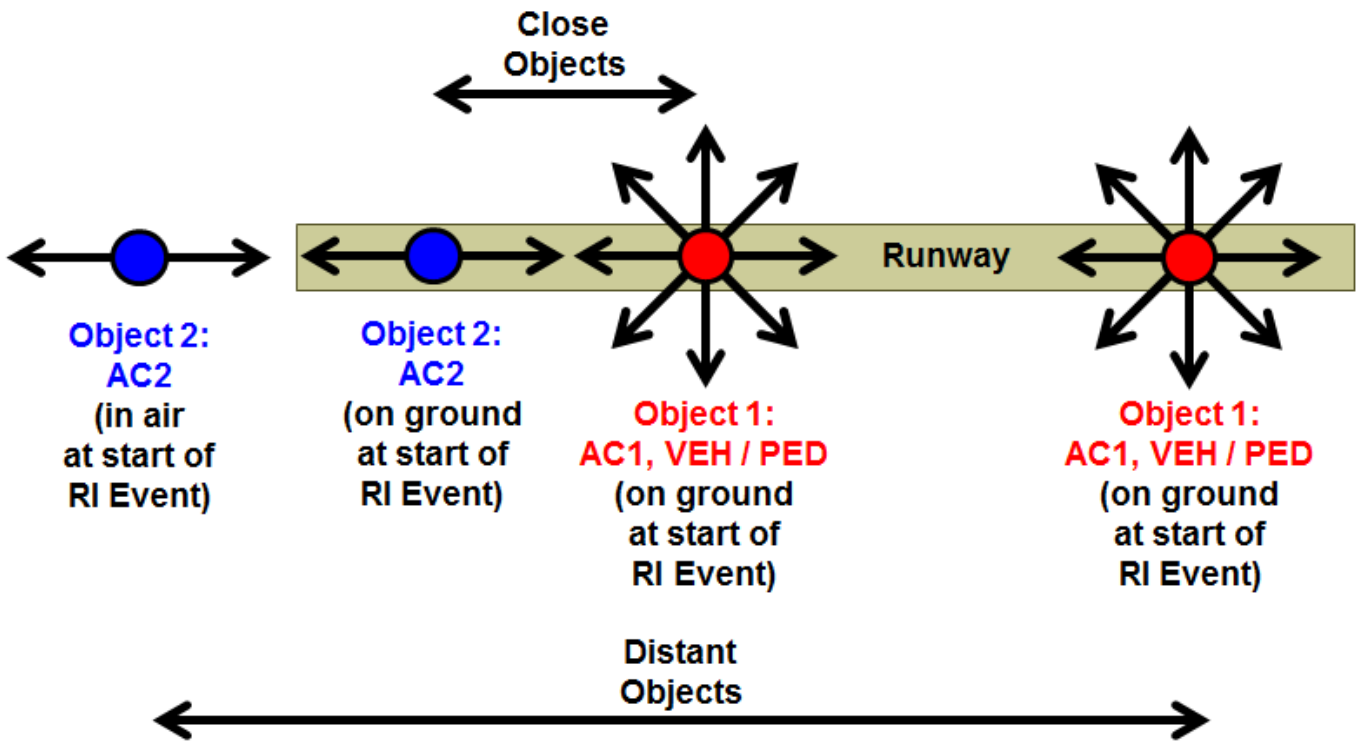

Figure 2. Geometric Considerations for the Runway Incursion Preliminary Model. 